\title{
NEW DOUBLE INEQUALITIES FOR MATHIEU TYPE SERIES
}

\author{
ŽIVORAD TOMOVSKI
}

Abstract. In this paper using the trapezoidal quadrature rule, we established new double inequality, for Mathieu's series of following type:

$$
S(a, p, \alpha)=\sum_{n=1}^{\infty} \frac{2 n^{\alpha / 2}}{\left(n^{\alpha}+a^{2}\right)^{p+1}}, \text { where } a>0, p>0, \alpha>0 .
$$

As a corollary of this inequality the solution of the problem posed by Tomovski and Trenčevski in [7], for $\alpha=2$ and $p>0$ is completed.

\section{INTRODUCTION}

In [6] Feng Qi intorduced the series of following type

$$
S(a, p, \alpha)=\sum_{n=1}^{\infty} \frac{2 n^{\alpha / 2}}{\left(n^{\alpha}+a^{2}\right)^{p+1}}, \text { where } a>0, \alpha>0 \text { and } p>0 .
$$

For $\alpha=2$ this series first was defined in [3] by Diananda.

Concerning the series $S(a, p, 2)$ in [3], P.H. Diananda proved the following Theorem:

Theorem A. If $a, p>0$ then

$$
S(a, p, 2)<\frac{1}{p a^{2 p}}
$$

For $\alpha=2, p=1$ the series $S(a, p, \alpha)$ was introduced in [5] by Mathieu. Thus the series:

$$
S(a, 1,2)=\sum_{n=1}^{\infty} \frac{2 n}{\left(n^{2}+a^{2}\right)^{2}}
$$

is called as Mathieu's series.

Mathieu (see [5]) obtained inequality for series $S(a, 1,2)$ which is a corollary of Theorem A for $p=1$.

Theorem B. If $a>0$, then

$$
S(a, 1,2)<\frac{1}{a^{2}}
$$

This theorem was refined by several authors (see [2],[3],[8]) but the best result was obtained recently by H. Alzer and J.L. Brenner. Namely they proved the following theorem.

Theorem C. [1] For all real numbers $a \neq 0$, we have:

$$
\frac{1}{a^{2}+1 /(2 \zeta(3))}<S(a, 1,2)<\frac{1}{a^{2}+1 / 6}
$$

1991 Mathematics Subject Classification. 33E20, 26D15.

Key words and phrases. inequality, integral expression, Mathieu's series, trapezoidal quadrature rule. 
The constants $1 /(2 \zeta(3))$ and $1 / 6$ are best possible, where $\zeta$ denotes the zeta function. On the other hand, Feng Qi in [6] established double inequality for series $S(a, 1, \alpha)$.

Theorem D. [6] Suppose a is a positive number, then for any positive real number $\alpha$, we have:

$$
\frac{1}{a^{2}+\frac{1}{2}}<S(a, 1, \alpha)<\frac{1}{a^{2}}
$$

In [7] we established double inequality for series $S(a, p, 2)$, when $a>0$ and $p \in \mathbb{N}$.

Theorem E. [7] If $a>0, p \in \mathbb{N}$, then

$$
\begin{aligned}
|S(a, p, 2)| & \leq \frac{2(2 a)^{-p}}{p ! a^{p+1}}\left[\sum_{k=0}^{\infty}(-1)^{k} \frac{(k \pi)^{p}}{\exp (k \pi / a)-1}+\sum_{k=0}^{\infty}(-1)^{k} \frac{\left(\left(k+\frac{1}{2}\right) \pi\right)^{p}}{\exp \left(\left(k+\frac{1}{2}\right) \frac{\pi}{a}\right)-1}\right] \\
& +\sum_{k=1}^{p} \frac{2(2 a)^{-2 p+k-1}}{k ! a^{k+1}}\left|\left(\begin{array}{c}
-(p+1) \\
p-k
\end{array}\right) \frac{1-k}{p-k+1}\right| \times\left[\sum_{j=0}^{\infty}(-1)^{j} \frac{(j \pi)^{k}}{\exp (j \pi / a)-1}\right. \\
& \left.+\sum_{j=0}^{\infty} \frac{\left(\left(j+\frac{1}{2}\right) \pi\right)^{k}}{\exp \left(\left(j+\frac{1}{2}\right) \pi / a\right)-1}\right]
\end{aligned}
$$

\section{The Integral EXPRESSiOn FOR SERIES $S(a, p, \alpha)$}

In this section, we shall establish an integral expression of $S(a, p, \alpha)$, where $a>0, p>0, \alpha>0$. This is an open problem, posed by Feng Qi in [6].

Theorem 1. For $a>0, p>0, \alpha>0$ the following integral expression of $S(a, p, \alpha)$ holds:

$$
S(a, p, \alpha)=\frac{2}{\Gamma(p+1)} \int_{0}^{\infty} x^{p} e^{-a^{2} x} g(x) d x
$$

where

$$
g(x)=\sum_{n=1}^{\infty} n^{\alpha / 2} e^{-n^{\alpha} x}
$$

Proof. Using the well-known formula:

$$
\frac{1}{t^{p+1}}=\frac{2}{\Gamma(p+1)} \int_{0}^{\infty} x^{p} e^{-x t} d x
$$

we obtain

$$
\frac{2 n^{\alpha / 2}}{\left(n^{\alpha}+a^{2}\right)^{p+1}}=\frac{2}{\Gamma(p+1)} \int_{0}^{\infty} x^{p} n^{\alpha / 2} e^{-\left(n^{\alpha}+a^{2}\right) x} d x .
$$

Applying the Cauchy integration test, we obtain that $\sum_{n=1}^{\infty} n^{\alpha / 2} e^{-n^{\alpha} x}$ is convergent for all $x>0$ and $\alpha>0$, i.e. $g(x)=\sum_{n=1}^{\infty} n^{\alpha / 2} e^{-n^{\alpha} x}$.

Thus 


$$
\begin{aligned}
S(a, p, \alpha) & =\frac{2}{\Gamma(p+1)} \int_{0}^{\infty} x^{p} e^{-a^{2} x}\left(\sum_{n=1}^{\infty} n^{\alpha / 2} e^{-n^{\alpha} x}\right) \\
& =\frac{2}{\Gamma(p+1)} \int_{0}^{\infty} x^{p} e^{-a^{2} x} g(x) d x .
\end{aligned}
$$

\section{Main Results}

Our main results are as follows

Theorem 2. If $a>0, p>0, \alpha>0$, then

$$
\begin{aligned}
\frac{2 \Gamma\left(\frac{1}{\alpha}+\frac{1}{2}\right) \Gamma\left(p-\frac{1}{\alpha}+\frac{1}{2}\right)}{\alpha \Gamma(p+1) a^{2 p-\frac{2}{\alpha}+1}} & -\frac{2 \Gamma\left(p+\frac{1}{2}\right)}{\sqrt{2 e} \Gamma(p+1) a^{2 p+1}}<S(a, p, \alpha)< \\
& <\frac{2 \Gamma\left(\frac{1}{\alpha}+\frac{1}{2}, 1\right) \Gamma\left(p-\frac{1}{\alpha}+\frac{1}{2}\right)}{\alpha \Gamma(p+1) a^{2 p-2 / \alpha+1}}+\frac{2 \Gamma\left(p+\frac{1}{2}\right)}{\sqrt{2 e} \Gamma(p+1) a^{2 p+1}}
\end{aligned}
$$

Proof. Let $f(x)=x^{\alpha / 2} e^{-x^{\alpha} t}, x>0, t>0$

Since $\int_{0}^{\infty} f(x) d x=\frac{\Gamma\left(\frac{1}{2}+\frac{1}{2}\right)}{\alpha t^{1 / \alpha+1 / 2}}$ and $\max _{x \in \mathbb{R}^{+}} f(x)=\frac{1}{\sqrt{2 e t}}$, applying the trapezoidal quadrature rule (see[4]):

$$
\int_{0}^{\infty} f(x) d x-\max _{x \in \mathbb{R}^{+}} f(x)<\sum_{n=1}^{\infty} f(n)<\int_{0}^{\infty} f(x) d x+\max _{x \in \mathbb{R}^{+}} f(x)-\int_{0}^{1} f(x) d x,
$$

we obtain

$$
\frac{\Gamma\left(\frac{1}{\alpha}+\frac{1}{2}\right)}{\alpha t^{\frac{1}{\alpha}+\frac{1}{2}}}-\frac{1}{\sqrt{2 e t}}<g(t)<\frac{\Gamma\left(\frac{1}{\alpha}+\frac{1}{2}, 1\right)}{\alpha t^{\frac{1}{\alpha}+\frac{1}{2}}}+\frac{1}{\sqrt{2 e t}},
$$

where $g$ is the function defined in Theorem 1 and $\Gamma(a, x)=\int_{x}^{\infty} e^{-t} t^{a-1} d t$.

Hence

$$
\begin{aligned}
& \frac{2 \Gamma\left(\frac{1}{\alpha}+\frac{1}{2}\right)}{\alpha \Gamma(p+1)} \int_{0}^{\infty} t^{p-\frac{1}{\alpha}-\frac{1}{2}} e^{-a^{2} t} d t-\frac{2}{\sqrt{2 e} \Gamma(p+1)} \int_{0}^{\infty} t^{p-\frac{1}{2}} e^{-a^{2} t} d t< \\
& S(a, p, \alpha)<\frac{2 \Gamma\left(\frac{1}{\alpha}+\frac{1}{2}, 1\right)}{\alpha \Gamma(p+1)} \int_{0}^{\infty} t^{p-\frac{1}{\alpha}-\frac{1}{2}} e^{-a^{2} t} d t+\frac{2}{\sqrt{2 e} \Gamma(p+1)} \int_{0}^{\infty} t^{p-\frac{1}{2}} e^{-a^{2} t} d t, \text { i.e. } \\
& \frac{2 \Gamma\left(\frac{1}{\alpha}+\frac{1}{2}\right) \Gamma\left(p-\frac{1}{\alpha}+\frac{1}{2}\right)}{\alpha \Gamma(p+1) a^{2 p-\frac{2}{\alpha}+1}}-\frac{2 \Gamma\left(p+\frac{1}{2}\right)}{\sqrt{2 e} \Gamma(p+1) a^{2 p+1}}<S(a, p, \alpha)< \\
& <\frac{2 \Gamma\left(\frac{1}{\alpha}+\frac{1}{2}, 1\right) \Gamma\left(p-\frac{1}{\alpha}+\frac{1}{2}\right)}{\alpha \Gamma(p+1) a^{2 p-2 / \alpha+1}}+\frac{2 \Gamma\left(p+\frac{1}{2}\right)}{\sqrt{2 e} \Gamma(p+1) a^{2 p+1}}
\end{aligned}
$$


As a corollary of this theorem we obtain new double inequalities for series $S(a, p, 2) a>0, p>0$, which complete the result of partially solved problem by Tomovski and Trenčevski (see [7]), when $p \in \mathbb{N}$.

Corollary. If $a, p>0$ then the following inequalities hold:

$$
\frac{2}{\Gamma(p+1)}\left(\frac{\Gamma(p)}{2 a^{2 p}}-\frac{\Gamma\left(p+\frac{1}{2}\right)}{\sqrt{2 e} a^{2 p+1}}\right)<S(a, p, 2)<\frac{2}{\Gamma(p+1)}\left(\frac{\Gamma(p)}{2 e a^{2 p}}+\frac{\Gamma\left(p+\frac{1}{2}\right)}{\sqrt{2 e} a^{2 p+1}}\right)
$$

Proof. By putting in Theorem 1, $\alpha=2$ the proof is completed.

\section{REFERENCES}

[1] H. Alzer, J.L. Brenner, On Mathieu's inequality, Journal of Mathematical Analysis and Applications 218, 607-610 (1998)

[2] P.H. Diananda, On Some Inequalities Related to the Mathieu's, Univ. Beograd, Publ. Electrotehn. fak. Ser. Mat. Fiz. 544-576 (1976), 77-80

[3] P.H. Diananda, Some inequalities Related to the Inequality of Mathieu, Math. Ann. 250, 95-98 (1980)

[4] N. L. Fernández, J. Prestin "Localization of the Spherical Gauss-Weierstrass Kernel, Constructive Theory of Functions", Varna 2002, pp.267-274

[5] E. Mathieu, "Traité de physique mathématique, VI-VII : Théorie de l' élasticité des corps solides", Gauthier Villars, Paris, 1890

[6] F. Qi, Inequalitis for Mathieu's series, RGMIA res. Rep. Coll. 4 (2001), no. 2, Art. 3, 187-197. Avaliable online at http://rgmia.vu.edu.au/v4n2.html

[7] Ž. Tomovski, K. Trenčevski, On an open problem of Bai-Ni Guo and Feng Qi, JIPAM, Vol. 4(2), (2003)

[8] C.L. Wang and X.H. Wang, A refinement of the Mathieu inequality, Univ. Beograd. Publ. Electrotehn. fak. Ser. Mat. Fiz. 716-734 (1981), 22-24

Institute of Mathematics, "St. Cyril and Methodius University, P.O. Box 162, Skopje, Macedonia

E-mail address: tomovski@iunona.pmf.ukim.edu.mk 